\title{
PHOTOVOLTAICS
}

\section{Flexible optoelectronics}

It is widely believed that the fabrication of foldable and bendable optoelectronics requires technologies such as organic polymer semiconductors or colloidal quantum dots, both of which can be deposited onto plastic substrates using solution-processing techniques.

It now turns out that foldable optoelectronics can also be made from conventional inorganic semiconductors such as $\mathrm{GaAs}$ and InP, thanks to a new innovative fabrication approach developed by John Rogers and co-workers at the University of Illinois at UrbanaChampaign and Northwestern University in the USA (Adv. Mater. doi:10.1002/ adma.201000591; 2010). Until recently, the deposition of such compound semiconductors onto a plastic substrate was thought to be impossible owing to their incompatibility with semiconductor fabrication technologies such as molecular beam epitaxy and metal organic chemical vapour deposition.

The researchers used an etch-andrelease scheme to fabricate red $(\sim 675 \mathrm{~nm})$ LEDs based on conventional GaAs-InGaP semiconductors on a thin sacrificial layer of AIGaAs deposited on a GaAs wafer. The clever part of the scheme is that the sacrificial AIGaAs layer can be etched away after fabrication, which allows the active semiconductor forming the LED to be transferred onto a thin plastic substrate. The researchers say that their approach produces brighter and more efficient LEDs than organic light emitters, while still enjoying the benefits of a flexible plastic substrate. A rather unexpected outcome is the extreme flexibility of the LEDs, which allegedly permits a bending radius as small as $0.7 \mathrm{~mm}$ - appreciably lower than the previous record of several millimetres using organic LED technology.

Fabrication of the device starts with the growth of microscale LEDs, each measuring $100 \mu \mathrm{m} \times 100 \mu \mathrm{m} \times 2.523 \mu \mathrm{m}$, using the etch-and-release scheme. The LEDs are then removed and transfer-printed as arrays onto a substrate of polyethylene terephthalate coated with polyurethane. An epoxy coating is applied, etching is performed and ohmic metals are then deposited to define the $\mathrm{n}$ - and $\mathrm{p}$-electrodes of the microscale LEDs. Electrical interconnection lines are formed through photolithographic patterning.

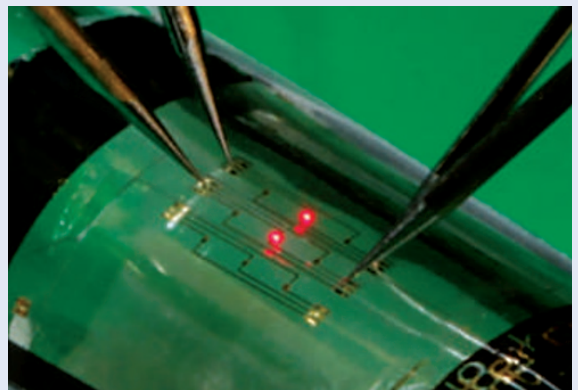

The final encapsulation process involves applying epoxy of varying thickness to different regions. The thickness of the encapsulation layer is incremented in steps during deposition to reduce the mechanical strain on the LED quantum wells when the substrate is folded or flexed.

It is suggested that the development of these highly flexible LED arrays, which have superior optical performance and mechanical properties to organic LEDs, may prove useful for creating flexible display technologies.

SONIA SHAHI

\section{SILICON PHOTONICS}

\section{Nonlinear optics in the mid-infrared}

Carefully designed nanophotonic silicon waveguides, when pumped at long wavelengths to avoid inherent losses, are opening the door to useful nonlinear processes in the mid-infrared.

\section{Bahram Jalali}

\section{$\mathrm{S}$} ilicon photonics is now a thriving community and a blossoming business thanks to its compatibility with the manufacturing infrastructure of silicon electronics. Owing to the loss characteristics of optical fibre, today's data interconnects exploit the near-infrared (IR) region of the electromagnetic spectrum at around $1,550 \mathrm{~nm}$, where fibre losses are lowest $\left(0.1-0.2 \mathrm{~dB} \mathrm{~km}^{-1}\right)$. However, silicon still suffers from an inherent problem at these wavelengths: a strong loss mechanism that 'kicks in' under high-power pumping. This tendency of silicon to become lossy at high intensities in the near-IR has been the major hurdle in creating practical optical amplifiers and wavelength converters - fundamental building blocks of data communication networks - in silicon photonics, as such devices require powerful pump sources to operate.

Although silicon is normally transparent and thus exhibits low propagation losses at near-IR wavelengths, at high intensities it begins to absorb light because of two-photon absorption (TPA), in which two photons can 'cooperate' to excite an electron out of the valence band and into the conduction band (Fig. 1). TPA creates a population of free carriers that can also absorb light through free-carrier absorption ${ }^{1-3}$. As silicon has an indirect electronic band structure, its intrinsic recombination rate of free carriers is low only $10^{3}-10^{6}$ per second (depending on its purity) - compared with approximately $10^{9}$ per second in direct-band structure semiconductors such as GaAs. Consequently, the free-carrier population quickly builds up at high light intensities, resulting in significant optical loss.

This problem dramatically reduces when the wavelength of the incident light exceeds $\sim 2,200 \mathrm{~nm}$ (refs 4,5), which is the threshold for TPA to occur (Fig. 1) 